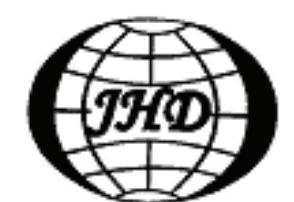

www.sciencedirect.com/ science/journal/10016058

\title{
ELECTROHYDRODYNAMIC DISPERSION OF DEFORMABLE AERO- SOLS IN THE PRESENCE OF AN ELECTRIC FIELD AND CHEMICAL REACTION USING TAYLOR DISPERSION MODEL*
}

\author{
RUDRAIAH N., DEVARAJU N. \\ UGC-CAS in Fluid Mechanics, Department of Mathematics, Bangalore University, Bangalore - 560 001, India \\ National Research Institute for Applied Mathematics (NRIAM), Bangalore - 560 070, India, \\ E-mail: rudraiahn@hotmail.com \\ NG Chiu-On \\ Department of Mechanical Engineering, The University of Hong Kong, Hong Kong, China
}

(Received December 3, 2010, Revised January 16, 2011)

\begin{abstract}
Under the effects of electric field and chemical reaction, the problem of dispersion of aerosols in a poorly conducting fluid in a channel is solved analytically using the mixture theory together with a regular perturbation technique. It is shown that the aerosols are dispersed relative to a plane moving with the mean speed of atmospheric fluid as well as the mean speed of agglomeration of aerosol with a relative diffusion coefficient, called the Taylor dispersion coefficient. This coefficient is numerically computed and the results reveal that it increases with an increase in the electric number, but decreases with increasing porous parameter. The physical explanations for the phenomena are given in this article.
\end{abstract}

Key words: dispersion, aerosols, electrohydrodynamics, mixture theory

\section{Introduction}

This article deals with the effects of electric field and chemical reaction on the dispersion of deformable aerosols in a poorly conducting atmospheric fluid flowing in a channel bounded by porous layers using Taylor's model ${ }^{[1]}$.

Electric fields are used extensively in different industrial problems, particularly in those concerned with chemical, electrical, electronic and drug industries for various separation processes. Waterman ${ }^{[2]}$ reviewed the process of using electric fields to improve coalescence and found these techniques to be quite effective in the removal of water from oil. Williams and Bailey ${ }^{[3]}$ examined coalescence of poorly conducting drops in the presence of an elec-

* Project supported by the Department of Science of Technology of India (Grant No. SR/S4-AS-237/2004-05), the Research Grants Council of the Hong Kong Special Administrative Region, China, (Grant Nos. HKU 715609E, HKU 715510E).

Biography: RUDRAIAH N. (1932-), Male, Ph. D., Professor Corresponding author: NG Chiu-On, E-mail: cong@hku.hk trical field both theoretically and experimentally. Schmidt ${ }^{[4]}$ performed experiments with different aerosols and observed that the application of an electric field had the effect of reducing sedimentation time. A detailed calculation of the aerosol particles and size distribution suggested that this phenomenon of reduction of sedimentation time is due to the electric field enhancing aerosol particle coalescence.

Studies of predicting the collision frequencies of settling mechanisms have been carried out in the past. Also, the deposition of nano-particles under different conditions was investigated in the recent numerical studies by Sun et al. ${ }^{[5]}$, Gan et al. ${ }^{[6]}$, Yin and $\operatorname{Lin}^{[7]}$, and Liu and $\operatorname{Lin}^{[8]}$. Wang et al. ${ }^{[9]}$ used a trajectory analysis for estimating the aggregation rates and found that the electric fields can enhance the gravitational settling of charged particles.

Usually, when the Earth's local weather is fine, the electric field is about $180 \mathrm{Vm}^{-1}-280 \mathrm{Vm}^{-1}$ depending on the concentration of aerosols (see Jayaratne and Verma ${ }^{[10]}$ ). When the aerosols are in continuous deformation with relative motion resulting in particles colliding and coalescing to form larger 
particles where an electric field induces a dipole in uncharged aerosol particles, as explained by Rudraiah and Devaraju ${ }^{[11]}$, who showed that the charges induced on the closest sides of the neighboring particles are of opposite sign. These particles experience an attractive force which can eventually lead to the particles colliding causing agglomeration or coalescence. That is, the difference in number concentration of aerosols causes variation in electrical conductivity $\sigma$, which releases the charges forming distribution of charge density $\rho_{\varepsilon}$. These charges produce an induced electric field, $\boldsymbol{E}_{\text {ind }}$, known as concentration electric field. In addition, there may be an applied electric field, $\boldsymbol{E}_{a}$, due to embedded electrodes of different potentials at the boundaries. The total electric field $\boldsymbol{E}_{t}=\boldsymbol{E}_{\text {ind }}+\boldsymbol{E}_{a}$ produces not only a current density, $\boldsymbol{J}$, according to Ohm's law, but also an electric force $\rho_{e} \boldsymbol{E}_{t}$, which is computed using Maxwell's equations.

The motion of atmospheric fluid is usually turbulent due to large length scales which result in large Reynolds number. Therefore, to derive the required basic equations, we use the mechanism of mixture of aerosols and atmospheric turbulent fluid taking into account the combined effect of electric field, deformable aerosols and settling of large particles on primary as well as secondary admixture using the Reynolds averaging procedure supplemented with gradient diffusion model. The primary pollutants, directly injected into the atmosphere, are assumed to satisfy the general equation involving the relative velocity between aerosol and atmospheric fluid. The aerosol medium is assumed to be homogeneous, incompressible and isotropic in the presence of the body force. In the literature ${ }^{[11-14]}$, mixture theory has been used to derive the basic equations for an ordinary fluid in the absence of electric field through assuming the mixture of aerosol and atmospheric fluid as deformable porous media. The dispersion of aerosols and other Suspended Particulate Matter (SPM) in the presence of electric field in atmospheric turbulent fluid has not been given much attention in the literature. The study of it is the main objective of this article because of its importance in many applications discussed above. To achieve the objectives of this article, the required basic equations, relevant boundary conditions and the dimensionless form are given in Section 1 on mathematical formulation of this article "space". Analytical solutions of coupled partial differential equations are derived in Section 2 using a regular perturbation technique with a perturbation parameter $\varepsilon$. The Taylor dispersion coefficients for atmospheric fluid and also for aerosols are determined in this section. These dispersion coefficients, the velocity for atmospheric fluid $u^{f}$ and for aerosols $u^{s}$ are numerically computed and the results are represented graphically and important conclusions are given in Section 3.

\section{Mathematical formulation}

We consider a two-dimensional geometry as shown in Fig.1. It consists of flow through a symmetrical channel extended to infinity on both directions of the $x$-axis. The channel is filled with poorly conducting fluid saturated porous media regarded as a mixture of aerosol and atmospheric fluid bounded by permeable layers with embedded electrodes of different potentials at $y=0$ and $y=h$. The applied pressure gradient $\partial p / \partial x=G(t)$ produces an axially directed flow. For an infinite channel, we assume fully developed unidirectional flow as in Taylor $^{[1]}$ so that there is no $x$-dependence in any of the physical quantities except the pressure, electric potential and concentration of aerosols. The unidirectional flow is represented with velocities $q_{1}^{s}=u^{s}$ as the $x$-component of aerosol velocity and $q_{1}^{f}=u^{f}$ the $x$-component of atmospheric fluid velocity. Then the required momentum equations are given by

$$
\begin{array}{r}
\overline{\rho^{\beta}}\left(\frac{\partial \overline{q_{i}^{\beta}}}{\partial t}+\overline{q_{j}^{\beta}} \frac{\partial \overline{q_{i}^{\beta}}}{\partial x_{j}}\right)=-\phi^{\beta} \frac{\partial \bar{p}}{\partial x_{i}}+\left[X_{p}\left(\mu-\mu_{a}\right)+\right. \\
\left.\mu_{a}+\mu_{e}\right] \frac{\partial^{2} \overline{q_{i}^{\beta}}}{\partial x_{j} \partial x_{j}} \mp K\left(\overline{q_{i}^{s}}-\overline{q_{i}^{f}}\right)+\rho_{e} E_{t}
\end{array}
$$

These equations, following Rudraiah and Devaraju ${ }^{[1]}$, for the mixture of aerosol and atmospheric turbulent fluid with the assumptions stated above becomes

$$
\begin{aligned}
& \rho^{s} \frac{\partial u^{s}}{\partial t}=\mu_{s} \frac{\partial^{2} u^{s}}{\partial y^{2}}-\phi^{s} G-K\left(u^{s}-u^{f}\right)+\rho_{e} E_{x} \\
& \rho^{f} \frac{\partial u^{f}}{\partial t}=\mu_{f} \frac{\partial^{2} u^{f}}{\partial y^{2}}-\phi^{f} G+K\left(u^{s}-u^{f}\right)+\rho_{e} E_{x}
\end{aligned}
$$

where the superscript, $s$, represents solid phase (i.e., aerosol) and, $f$, the fluid phase (i.e., atmospheric poorly conducting fluid), $\mu_{s}=\mu+\mu_{e}$ is one of the turbulent Lamé constants, $\mu_{f}=\mu_{a}+\mu_{e}$ the effective viscosity (also called the Brinkman viscosity) of poorly conducting atmospheric turbulent fluid, $\mu$ the Lamé constant, $\mu_{e}$ the eddy viscosity, $K$ the linear drag coefficient that is the Darcy resistance offered by solid to fluid, $G$ the pressure gradient, 
$\varphi^{s}$ the aerosol volume fraction and $\varphi^{f}$ the fluid volume fraction. We make these equations dimensionless using

$\eta=\frac{y}{h}, t^{*}=\frac{t}{t_{0}}, u^{s^{*}}=\frac{\mu_{s}}{h^{2} G_{0}} u^{s}, u^{f^{*}}=\frac{\mu_{f}}{h^{2} G_{0}} u^{f}$,

$G^{*}=\frac{G}{G_{0}}, \quad E_{x}^{*}=\frac{E_{x}}{V / h}, \quad \rho_{e}^{*}=\frac{\rho_{e}}{\varepsilon_{0} V / h^{2}}, \quad \Phi^{*}=\frac{\Phi}{V}$,

$v^{f}=\frac{\mu_{f}}{\rho^{f}}, v^{s}=\frac{\mu_{s}}{\rho^{s}}$

where $h, G_{0}, V$ and $t_{0}$ are the characteristic length, pressure gradient, electric potential and time respectively and the asterisks $\left({ }^{*}\right)$ denote the dimensionless quantities.

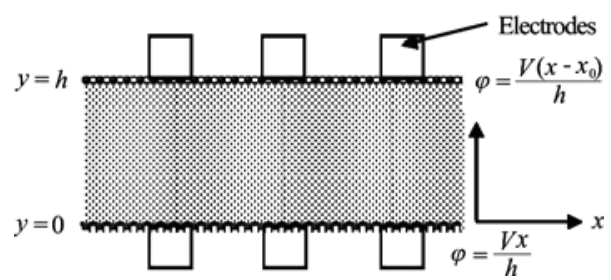

Fig.1 Physical configuration

Substituting Eq.(4) into Eqs.(2) and (3), simplifying and for simplicity neglecting the asterisks, we get

$\frac{\partial u^{s}}{\partial t}=R_{1} \frac{\partial^{2} u^{s}}{\partial \eta^{2}}-R_{1} \phi^{s} G-R_{2}\left(u^{s}-R_{3} u^{f}\right)+W_{e} \rho_{e} E_{x}$

$\frac{\partial u^{f}}{\partial t}=R_{4} \frac{\partial^{2} u^{f}}{\partial \eta^{2}}-R_{4} \phi^{s} G+R_{2} \lambda\left(u^{s}-R_{3} u^{f}\right)+W_{e} \rho_{e} E_{x}$

where $R_{i}(i=1$ to 4$)$ are dimensionless parameters defined by

$R_{1}=\frac{\mu_{s} t_{0}}{\rho^{s} h^{2}}, \quad R_{2}=\frac{K t_{0}}{\rho^{s}}, R_{3}=\frac{\mu_{s}}{\mu_{f}}, R_{4}=\frac{\mu_{f} t_{0}}{\rho^{f} h^{2}}$,

$\lambda=\frac{v^{f}}{v^{s}}, W_{e}=\frac{\varepsilon_{0} V^{2}}{\rho^{s} h^{2}\left(\bar{u}^{s}\right)^{2}}=\frac{\varepsilon_{0} V^{2}}{\rho^{f} h^{2}\left(\bar{u}^{f}\right)^{2}}$

The boundary conditions are the Saffman slip conditions of the form ${ }^{[15]}$ $\frac{\mathrm{d} u^{s}}{\mathrm{~d} \eta}= \pm \alpha_{1} \sigma_{p} u^{s}, \frac{\mathrm{d} u^{f}}{\mathrm{~d} \eta}= \pm \alpha_{2} \sigma_{p} u^{f}$ at $\eta=0$,

and 1 for $t>0$, respectively

where $\sigma_{p}=h / \sqrt{k}$ is the porous parameter, and $\alpha_{1}$ and $\alpha_{2}$ are the slip parameters. To find the electric force, $\rho_{e} E_{x}$, first we have to find the electric field $E_{x}$ and then the charge density $\rho_{e}$ using Maxwell's equations given by

Gauss's law:

$\frac{\partial E_{i}}{\partial x_{i}}=\frac{\rho_{e}}{\varepsilon_{0}}$

Faraday's law:

By neglecting induced magnetic field because $\sigma \ll 1$, and due to the absence of applied magnetic field, is

$\varepsilon_{i j k} \frac{\partial E_{i}}{\partial x_{j}}=0 \Rightarrow E_{i}=-\frac{\partial \Phi}{\partial x_{i}}$

Ampere's law:

$\varepsilon_{i j k} \frac{\partial H_{i}}{\partial x_{j}}=J_{i}+\rho_{e} q_{i}$

Solenoidal property of magnetic field:

$\frac{\partial H_{i}}{\partial x_{i}}=0$

These equations have to be supplemented with the equation of continuity of charges

$\frac{\partial \rho_{e}}{\partial t}+\frac{\partial\left(\rho_{e} q_{i}\right)}{\partial x_{i}}+\frac{\partial J_{i}}{\partial x_{i}}=0$

and using Ohm's law for a poorly conducting media $(\sigma \ll 1)$ and neglecting convection current $\rho_{e} q_{i}$ compared to conduction current, we denote $D_{i}=\varepsilon_{0} E_{i}$, $J_{i}=\sigma E_{i}$. From these, while using the assumption $\sigma \ll 1$, Eq.(13) becomes

$\frac{\partial \rho_{e}}{\partial t}+\sigma \frac{\partial E_{i}}{\partial x_{i}}+E_{i} \frac{\partial \sigma}{\partial x_{i}}=0 \quad\left(\because \frac{\partial q_{i}}{\partial x_{i}}=0\right)$

Furthermore, using Eqs.(9) and (10), and taking $\sigma=\sigma(\eta)$, we get

$\frac{\partial^{2} \Phi}{\partial \eta^{2}}+\frac{1}{\sigma} \frac{\partial \Phi}{\partial \eta} \frac{\partial \sigma}{\partial \eta}=0$ 
with the boundary conditions due to potentials $\Phi=V x / h \quad$ at $\quad y=0$ and $\Phi=V\left(x-x_{0}\right) / h$ at $y=h$ due to embedded electrodes as shown in Fig.1. Making $\Phi$ dimensionless using $V$, the boundary conditions are

$\Phi=x-x_{0}$ at $\eta=1$

$\Phi=x$ at $\eta=0$

Since $\sigma \ll 1$, perturbation on it is negligible and hence $\sigma$ depends on the basic concentration $C_{b}$. Make $\sigma$ dimensionless using $\sigma_{0}$, and use $C_{b}$ the basic concentration obtained by solving $\mathrm{d}^{2} C_{b} / \mathrm{d} \eta^{2}=$ 0 satisfying $C_{b}=C_{0}$ at $\eta=0$ and $C=C_{1}$ at $\eta=1$, in the form $C_{b}=\left(C_{1}-C_{0}\right) \eta+C_{0}$. Then $\sigma$ is given by

$\sigma=1+\alpha \eta \approx \mathrm{e}^{\alpha \eta} \quad($ since $\alpha \ll 1)$

where $\alpha=\alpha_{h} \Delta C, \Delta C=C_{1}-C_{0}$. Eq.(15), using Eq.(17), becomes

$$
\frac{\mathrm{d}^{2} \Phi}{\mathrm{d} \eta^{2}}+\alpha \frac{\mathrm{d} \Phi}{\mathrm{d} \eta}=0
$$

The solution of Eq.(18), using the boundary condition given in Eq.(16), is

$$
\Phi=x-\frac{x_{0}\left(1-\mathrm{e}^{-\alpha \eta}\right)}{1-\mathrm{e}^{-\alpha}}
$$

Substituting Eq.(19) into $E_{x}$ and $\rho_{e}$, we get

$$
E_{x}=-\frac{\partial \Phi}{\partial x}=-1 \text { and } \rho_{e}=-\frac{\partial^{2} \Phi}{\partial \eta^{2}}=\frac{x_{0} \alpha^{2} \mathrm{e}^{-\alpha \eta}}{1-\mathrm{e}^{-\alpha}}
$$

If $C$ is the concentration of aerosols in the atmosphere, and diffuses in the atmospheric turbulent fluid of unidirectional flow given by Eqs.(2) and (3), then $C$, with the first-order chemical reaction, satisfies the equation

$$
\frac{\partial C}{\partial t}+u^{\beta} \frac{\partial C}{\partial x}=D_{e}\left(\frac{\partial^{2} C}{\partial x^{2}}+\frac{\partial^{2} C}{\partial y^{2}}\right)-k_{1} C
$$

We study the electrohydrodynamic dispersion of aerosols by the atmospheric fluid and vice versa using Taylor's model, in the next section.

\section{Electrohydrodynamic dispersion of aerosols}

The required basic equations and the corresponding boundary conditions are given by Eqs.(5) to (16). These equations are coupled linear Partial Differential Equations (PDE). Although it is possible to decouple these equations using a suitable operator, the resulting PDE becomes higher order and cumbersome and the required boundary conditions have to be extrapolated. Therefore, we avoid this process and use a regular perturbation technique choosing $\varepsilon=R_{2} R_{3}$ ( $\left.=K \mu_{s} t_{0} /\left(\rho^{s} \mu_{f}\right)\right)$ to be small as a perturbation parameter. This assumption $K \mu_{s} / \rho^{s} \ll \mu_{f} / t_{0}$ is valid in our study of quasi-steady flow obtained following Taylor ${ }^{[1]}$, where $t_{0}$ is very large and $\mu_{f}$, the viscosity of atmospheric fluid, is small. In this technique, we look for solutions of Eqs.(5) and (6) in the form

$u^{f}=u_{0}^{f}+\varepsilon u_{1}^{f}+\ldots, \quad u^{s}=u_{0}^{s}+\varepsilon u_{1}^{s}+\ldots$

Substituting Eqs.(22) and (20) into Eqs.(5) and (6) and assuming the normal mode solutions of the form

$\left\{u_{i}^{f}(y, t), u_{i}^{s}(y, t), G(t)\right\}=\left\{u_{i}^{f}(y), u_{i}^{s}(y), G_{0}\right\} \mathrm{e}^{-n t}$

where $i=0,1, \ldots$, and on simplifying them, we get

$$
\begin{aligned}
& \frac{\mathrm{d}^{2} u_{0}^{s}}{\mathrm{~d} \eta^{2}}-a_{0}^{2} u_{0}^{s}=\phi^{s}-\frac{W_{e}}{R_{1}} \mathrm{e}^{-\alpha \eta} \\
& \frac{\mathrm{d}^{2} u_{0}^{f}}{\mathrm{~d} \eta^{2}}+b_{0}^{2} u_{0}^{f}=\phi^{f}-\frac{\lambda R_{2}}{R_{4}} u_{0}^{s}-\frac{W_{e}}{R_{4}} \mathrm{e}^{-\alpha \eta}
\end{aligned}
$$

$\frac{\mathrm{d}^{2} u_{1}^{s}}{\mathrm{~d} \eta^{2}}-a_{0}^{2} u_{1}^{s}=-\frac{u_{0}^{f}}{R_{1}}$

$\frac{\mathrm{d}^{2} u_{1}^{f}}{\mathrm{~d} \eta^{2}}+b_{0}^{2} u_{1}^{f}=\frac{\lambda}{R_{4}} u_{0}^{f}-\frac{\lambda R_{2}}{R_{4}} u_{1}^{s}$

where $a_{0}^{2}=\left(R_{2}-n\right) / R_{1}$ and $b_{0}^{2}=n / R_{4}$. The boundary conditions are the Saffman slip conditions ${ }^{[14]}$ as given in Eq.(8), which become

$\frac{\mathrm{d} u_{i}^{s}}{\mathrm{~d} \eta}= \pm \alpha_{1} \sigma_{p} u_{i}^{s}, \frac{\mathrm{d} u_{i}^{f}}{\mathrm{~d} \eta}= \pm \alpha_{2} \sigma_{p} u_{i}^{f}$ at $\eta=0$,

and 1 for $t>0$, respectively 
Similarly we can get equations for $i>1$, but we restrict only to $i=0$ and 1 . The solutions of Eqs.(24) to (27), satisfying the condition given by Eq.(28), are

$$
\begin{aligned}
u_{0}^{s}= & c_{1} \cosh a_{0} \eta+c_{2} \sinh a_{0} \eta-\frac{W_{e} \mathrm{e}^{-\alpha \eta}}{R_{1}\left(\alpha^{2}-a_{0}^{2}\right)}-\frac{\phi^{s}}{a_{0}^{2}} \\
u_{0}^{f}= & \Delta_{11} \cos b_{0} \eta+\Delta_{12} \sin b_{0} \eta+\Delta_{13} \cosh a_{0} \eta+ \\
& \Delta_{14} \sinh a_{0} \eta+\Delta_{15} \mathrm{e}^{-\alpha \eta}+\Delta_{16} \\
u_{1}^{s}= & \left(\Delta_{17}+\Delta_{18} \eta\right) \sinh a_{0} \eta+\left(\Delta_{19}+\Delta_{20} \eta\right) \cosh \eta+ \\
& \Delta_{21} \sin b_{0} \eta+\Delta_{22} \cos b_{0} \eta+\Delta_{23} \mathrm{e}^{-\alpha \eta}+\Delta_{24} \\
u_{1}^{f}= & \left(\Delta_{25}+\Delta_{26} \eta\right) \cos b_{0} \eta+\left(\Delta_{27}+\Delta_{28} \eta\right) \sin b_{0} \eta+ \\
& \left(\Delta_{29}+\Delta_{30} \eta\right) \cosh a_{0} \eta+\left(\Delta_{31}+\Delta_{32} \eta\right) \sinh a_{0} \eta+ \\
& \Delta_{33} \mathrm{e}^{-\alpha \eta}+\Delta_{34}
\end{aligned}
$$

where the coefficients, $\Delta_{1 j}(j=1, \ldots, 9), \quad \Delta_{2 j}(j=$ $0, \ldots, 9), \Delta_{3 j}(j=0, \ldots, 4)$, are given in the appendix of this article.

From Eq.(22), using Eqs.(29) to (32), we get

$$
\begin{aligned}
u^{s}= & u_{0}^{s}+\varepsilon u_{1}^{s}=\left[\left(a_{11}+a_{12} \eta\right) \cosh a_{0} \eta+\left(a_{13}+a_{14} \eta\right) \cdot\right. \\
& \left.\sinh a_{0} \eta+a_{15} \sin b_{0} \eta+a_{16} \cos b_{0} \eta+a_{17} \mathrm{e}^{-\alpha \eta}+a_{18}\right]
\end{aligned}
$$

$$
\begin{aligned}
u^{f}= & u_{0}^{f}+\mathcal{E} u_{1}^{f}=\left[\left(b_{11}+b_{12} \eta\right) \cos b_{0} \eta+\left(b_{13}+b_{14} \eta\right) \bullet\right. \\
& \sin b_{0} \eta+\left(b_{15}+b_{16} \eta\right) \cosh a_{0} \eta+\left(b_{17}+b_{18} \eta\right) \cdot \\
& \left.\sinh a_{0} \eta+b_{19} \mathrm{e}^{-\alpha \eta}+b_{20}\right]
\end{aligned}
$$

where the coefficients $a_{1 j}(i=1, \ldots, 8)$ and $b_{1 j}(i=$ $1, \ldots, 9), b_{20}$ are given in the appendix.

To find the concentration distribution, we assume, following Taylor ${ }^{[1]}$, the longitudinal diffusion is much less than the transverse diffusion, which implies $\partial^{2} C / \partial x^{2} \ll \partial^{2} C / \partial y^{2}$. Furthermore, we assume that though there is a small longitudinal gradient of $C$ along the flow, advection generates a small amount of aerosol across a section of the flow which moves with the mean speed. In other words, this small transport and the small longitudinal concentration gradient must be proportional to one another. Thus the combined effect of longitudinal advection and transverse diffusion is to disperse the aerosol longitudinally relative to a frame moving at the mean speed of flow by a mechanism which obeys the same law as an ordinary one-dimensional diffusion relative to fluid at rest. Then Eq.(19) takes the form

$$
\frac{\partial C}{\partial t}+u^{\beta} \frac{\partial C}{\partial x}=D_{e} \frac{\partial^{2} C}{\partial y^{2}}-k_{1} C
$$

In this article, we consider two situations, one is for the advection of aerosols by the atmospheric fluid, and the other for the advection of atmospheric fluid by agglomeration, the solid phase of aerosol. These are discussed as follows.

\subsection{Advection by atmospheric fluid}

In this case, by denoting $\beta=f$, Eq.(35) takes the form

$$
\frac{\partial C}{\partial t}+u^{f} \frac{\partial C}{\partial x}=D_{e} \frac{\partial^{2} C}{\partial y^{2}}-k_{1} C
$$

Making this equation dimensionless using the scales

$$
C^{*}=\frac{C}{C_{0}}, t^{*}=\frac{t}{t_{0}}, \eta=\frac{y}{h}, \xi=\frac{x-\bar{u}^{f} t}{L}, u^{f *}=\frac{u^{f}}{U}
$$

where $c_{0}$ is the initial slug, $L$ is the characteristic length along this flow direction, $U=h^{2} G_{0} / \mu_{f}$ is the characteristic velocity and all other scales are the same as those defined in Eq.(4) and

$$
\bar{u}^{f}=\int_{0}^{1} u^{f} \mathrm{~d} \eta=F
$$

is the average fluid velocity, where

$$
\begin{aligned}
F= & \left(\frac{b_{11}+b_{12}}{a_{0}}-\frac{b_{14}}{a_{0}^{2}}\right) \sinh a_{0}-\left(\frac{b_{13}+b_{14}}{a_{0}}-\frac{b_{12}}{a_{0}^{2}}\right) \\
& \cosh a_{0}+\left(\frac{b_{15}+b_{16}}{b_{0}}-\frac{b_{18}}{b_{0}^{2}}\right) \sin b_{0}- \\
& \left(\frac{b_{17}+b_{18}}{b_{0}}-\frac{b_{16}}{b_{0}^{2}}\right) \cos b_{0}+\left(\frac{b_{12}}{a_{0}^{2}}-\frac{b_{13}}{a_{0}}+\frac{b_{16}}{b_{0}^{2}}+\right. \\
& \left.\frac{b_{17}}{a_{0}}\right)+b_{19}
\end{aligned}
$$

and for simplicity neglecting the asterisks, we get 
$\frac{1}{t_{0}} \frac{\partial C}{\partial t}+\frac{w^{f}}{L} \frac{\partial C}{\partial \xi}=\frac{D}{h^{2}} \frac{\partial^{2} C}{\partial \eta^{2}}-k_{1} C$

where

$$
\begin{aligned}
w^{f}= & u^{f}-\bar{u}^{f}=\left(b_{11}+b_{12} \eta\right) \cosh a_{0} \eta+\left(b_{13}+b_{14} \eta\right) \bullet \\
& \sinh a_{0} \eta+\left(b_{15}+b_{16} \eta\right) \cos b_{0} \eta+\left(b_{17}+b_{18} \eta\right) \bullet \\
& \sin b_{0} \eta-f_{0}
\end{aligned}
$$

where $f_{0}=F-b_{19}$, and $b_{1 j}(i=1, \ldots, 9)$ are constants given in the appendix.

Following Taylor ${ }^{[1]}$, we assume the partial equilibrium at any cross-section of the layer and obtain concentration as a function of $y$ by approximating Eq.(36) in the form

$$
\frac{\partial^{2} C}{\partial \eta^{2}}-\beta_{1}^{2} C=D_{1} w^{f}
$$

where $D_{1}=\left(h^{2} / D L\right)(\partial C / \partial \xi)$ and $\beta_{1}^{2}=h^{2} k / D_{e}$ is the reaction rate parameter. For compatibility with the atmospheric layer we solve this equation for permeable to concentration given by

$$
C=1 \text { at } \eta=0, \frac{\partial C}{\partial \eta}=0 \text { at } \eta=1
$$

The solution of Eq.(42), after using Eqs.(41) and (43), is

$$
\begin{aligned}
C= & \left(c_{1} \cosh \beta_{1} \eta+c_{2} \sinh \beta_{1} \eta+D_{1}\right)\left(e_{11}+e_{12} \eta\right) \cdot \\
& \cosh a_{0} \eta+\left(e_{13}+e_{14} \eta\right) \sinh a_{0} \eta-\left(e_{15}+e_{16} \eta\right) \bullet \\
& \cos b_{0} \eta-\left(e_{17}+e_{18} \eta\right) \sin b_{0} \eta+\frac{f_{0}}{\beta_{1}^{2}}
\end{aligned}
$$

where $c_{1}, c_{2}$ and $e_{1 i}(i=1, \ldots, 7)$ are constants and are given in the appendix. The volumetric rate at which the aerosol is transported across a section of a layer of unit breadth is

$$
M=\int_{0}^{1} C w^{f} \mathrm{~d} \eta
$$

Hence, using Eqs.(44) and (45), performing the integration and simplifying the result, we get
$M=\frac{N_{1}^{f} h^{2}}{L D_{e}} \frac{\partial C}{\partial \xi}+1$

where $N_{1}^{f}=\int_{0}^{1} D_{1}^{-1}(C-1) w^{f} \mathrm{~d} \eta$. Following Taylor ${ }^{[1]}$, we assume that the variation of $C$ with $y$ is small compared to the longitudinal direction and if $C_{m}$ is the mean concentration over a section, then $\partial C / \partial \xi$ is indistinguishable from $\partial C_{m} / \partial \xi$ so that Eq.(46) can be written as

$M=\frac{N_{1}^{f} h^{2}}{L D_{e}} \frac{\partial C_{m}}{\partial \xi}+1$

This shows that $C_{m}$ will be dispersed relative to plane moving with the mean velocity $\bar{u}^{f}$ exactly as though it is being diffused by a process which obeys the same law as molecular diffusion but with a relative diffusion coefficient

$$
D^{f}=-N_{1}^{f} \frac{h \bar{u}^{f}}{D_{e}}=N^{f} P e^{f}
$$

called the Taylor diffusion coefficient for the mixture of deformable agglomeration and atmospheric fluid, $P e^{f}=h \bar{u}^{f} / D_{1}$ is the Peclet number and

$N^{f}=-N_{1}^{f}=-\int_{0}^{1}\left(\frac{C-1}{D_{1}}\right) w^{f} \mathrm{~d} \eta$

and $w^{f}$ is given in Eq.(41). This is integrated using Eqs.(44) and (41). The fact that no material is lost in the process is expressed by the continuity equation for $C_{m}$ namely

$\frac{\partial M}{\partial \xi}=-\frac{1}{L} \frac{\partial C_{m}}{\partial t}$

where the time derivative pertains to a point at which $\xi$ is fixed. Equation (47), using Eqs.(49) and (50), takes the form

$\frac{\partial C_{m}}{\partial t}=D^{f} \frac{\partial^{2} C_{m}}{\partial \xi^{2}}$

This equation represents the longitudinal dispersion of deformable agglomeration in the atmospheric fluid, dispersed with the dispersion coefficient $D^{f}$ given by Eq.(48). This is computed for different values of 
the electric number $W_{e}$, Reynolds number $R e$, Peclet number $P e$ and reaction rate parameter $\beta_{1}$, and the results are depicted graphically and are discussed in Section 3.

\subsection{Advection by agglomeration of aerosol}

We also note that the advection of atmospheric fluid by agglomeration can also be discussed through the similar analysis in Section 2.1 by substituting $\beta=s$ in Eq.(35). Following the same procedure as explained in Subsection 2.1, we get

$D^{s}=P e^{s} N^{s}$

where $P e^{s}=h \bar{u}^{s} / D_{m}$ and

$$
N^{s}=-\int_{0}^{1}\left(\frac{C-1}{D_{1}}\right) w^{s} \mathrm{~d} \eta
$$

As in Eq.(49), this is integrated using $w^{s}$ and $C-1$, and $D^{s}$ is computed for different values of the electric number $W_{e}$, Reynolds number $R e$, Peclet number $P e$ and the reaction rate parameter $\beta_{1}$, the results are depicted graphically and are discussed in the next section.

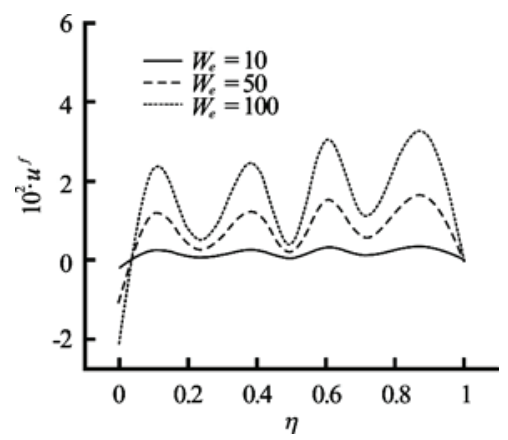

Fig.2 Velocity profile for atmospheric fluid for different values of electric number $W_{e}$

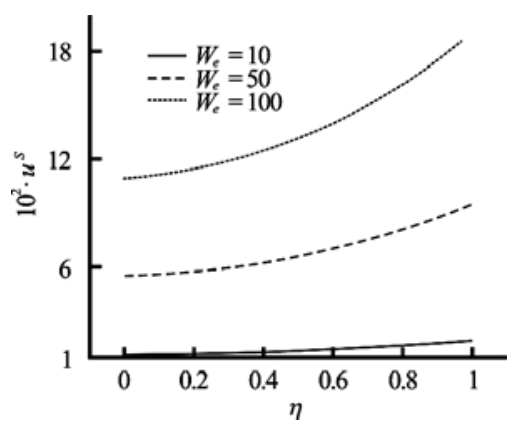

Fig.3 Velocity profiles of aerosols for different values of electric number $W_{e}$

\section{Results and discussions}

One of the necessary tasks associated with industrialization is to achieve progress in the removal of solid or liquid particles from gases discharged to the environment. Design of optimized pollution control devices becomes increasingly important towards achieving that goal. Fibrous filters are effective in the removal of submicrometer aerosol particles but their application is limited to low concentrations of aerosol dust in air. On the other hand, cyclones are efficient only at high gas flow rates and for larger particles. Cyclones, very common devices though, are still objects of intensive research because of their wide range of applications. In the case of smaller aerosol particulates an increase in the collection efficiency of particles in the turbulent flow is necessary. An application of an externally applied electric field in a turbulent flow assisting the inertial removal effect should result in increased cleaning effectiveness, in particular for small particles of poorly conducting materials. A standard design cyclone, with a tangential inlet in which an electric field is generated in the space between an external wall and the central outlet channel is considered. The dispersion of large size deformable aerosols mixed with a poorly conducting atmospheric fluid is discussed in this article analytically using Taylor's model valid asymptotically for large time with the objective of understanding the effect of electric field on the dispersion of atmospheric aerosols because of its importance in the applications mentioned above. The dispersion of deformable agglomeration in quasi-steady flow is discussed in Section 2. The equations governing the flow for atmospheric fluid, poorly conducting fluid and for deformable agglomeration obtained under the assumption of fully developed flow are the coupled partial differential Eqs.(5) and (6). These equations are solved analytically using the regular perturbation technique given by Eq.(22) with $\varepsilon=K \mu_{s} t_{0} /$ $\left(\rho^{s} \mu_{f}\right) \ll 1$. This assumption is valid in our study of quasi-steady flow as considered in Rudraiah and $\mathrm{Ng}^{[16]}$. Using these approximations and following the analysis of Taylor we obtained the solutions analytically. Using these solutions the dispersion coefficient $D^{\beta}$ is determined as given by Eq.(48) for advection by atmospheric fluid $(\beta=f)$ and by Eq.(52) for advection by agglomeration $(\beta=s)$. The results are represented graphically. Figures 2 and 3 represent the effect of electric number on the velocity profiles of atmospheric fluid and aerosols. From these figures we find that $u^{f}$ increases with $W_{e}$, because electric field induces a small scale turbulence but decreases with an increase in $\sigma_{p}$ because the permeability dampens the velocity profile. Figure 4 represents the effect of porous parameter on the dispersion of aerosols in an atmospheric poorly 


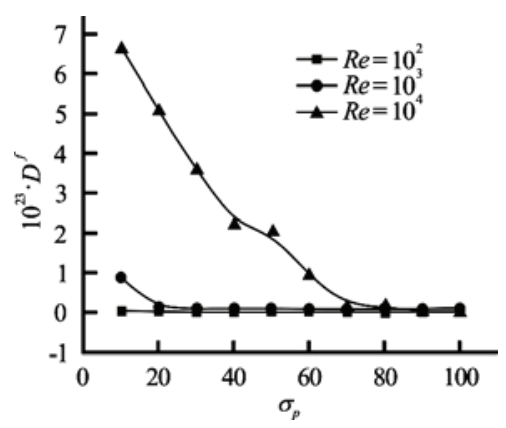

Fig.4 Dispersion coefficient $D^{f}$ versus porous parameter for different $R e$

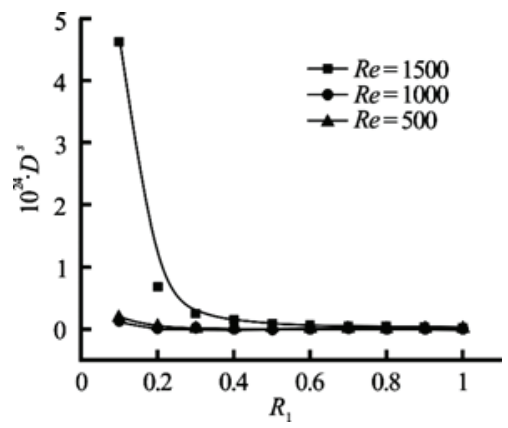

Fig.5 Dispersion coefficient $D^{s}$ versus deformation parameter for different $R e$

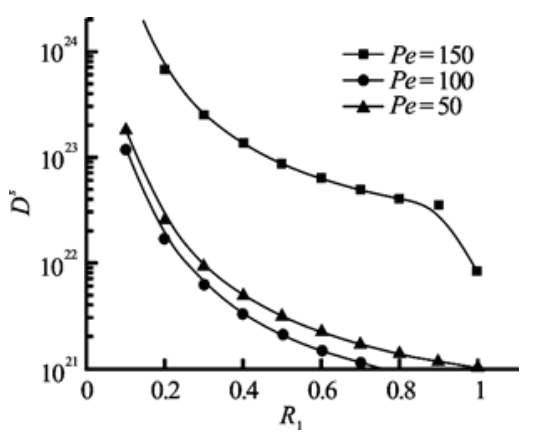

Fig.6 Dispersion coefficient $D^{s}$ versus deformation parameter for different $\mathrm{Pe}$

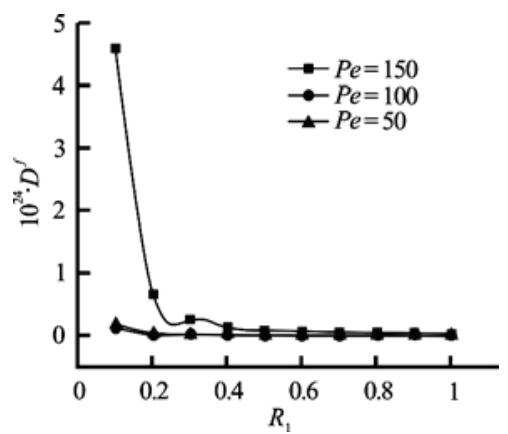

Fig.7 Dispersion coefficient $D^{f}$ versus deformation parameter for different $\mathrm{Pe}$

conducting fluid for different values of the Reynolds number $R e$ and it shows that the dispersion coefficient $D^{f}$ decreases with an increase in porous parameter because of its dampening effect but increases with an increase in the Reynolds number. Figures 5 and 6 show the effect of deformation parameter on the dispersion coefficient $D^{s}$ for different values of the Reynolds number and this figure shows that the $D^{s}$ decreases for small values of $R_{1}$ up to 0.2 and for higher values from 0.25 it remains uniform. It also reveals that increase in $R e$ slightly increases for the values of $R_{1}$ up to 0.5 and $D^{f}$ remains uniform for $R_{1}>0.5$, Figure 7 shows that $D^{f}$ decreases with an increase in $R_{1}$ up to 0.5 and also decreases with an increase in $P e$ up to $R_{1}=0.5$ and then remain uniform with respect to increase in $R_{1}$ and $P e$. Figure 6 shows that $D^{s}$ decreases with an increase in $R_{1}$ and for $R_{1}>0.7$ it tends to unity. However, $D^{s}$ decreases with an increase in $P e$ up to 100 and tends to $10^{21}$ at $R_{1}=1$ and for $P e>100$ it increases. Figure 8 shows that the $D^{f}$ increases with an increase in $W_{e}$ for $P e=150$. Similarly, Fig.9 shows that $D^{f}$ increases with an increase in reaction rate parameter $\beta_{1}$ for $W_{e}=50$ and $R e=10^{3}$ and reaches the maximum value $4.5 \times 10^{24}$ for $\beta_{1}=40$.

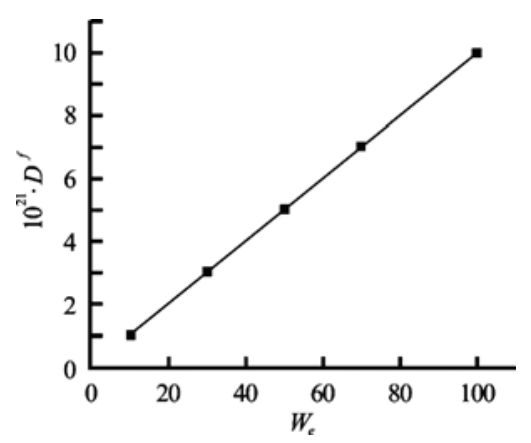

Fig.8 Dispersion coefficient $D^{f}$ versus electric number $W_{e}$

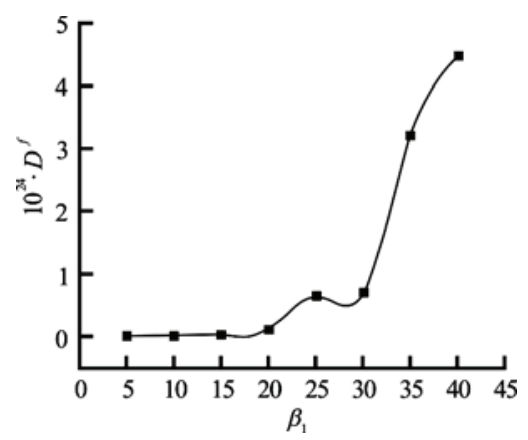

Fig.9 Dispersion coefficient $D^{f}$ versus reaction rate parameter 
[9] WANG H., ZENG S. and LOEWENBERG M. et al. Particle aggregation due to combined gravitational and electrophoretic motion[J]. J. Colloid Interface Sci., 1997, 187(1): 213-220.

\section{Conclusion}

In this article we have investigated the effect of electric field and the first-order chemical reaction on the dispersion of aerosols in a channel bounded by porous layers using Taylor's model. Analytical solutions for velocities and concentration distributions are obtained using a regular perturbation technique. It is shown that the aerosols are dispersed relative to a plane moving with the mean speed of atmospheric fluid as well as the mean speed of agglomeration of aerosol with a relative diffusion coefficient, called Taylor dispersion coefficient. This dispersion coefficient is numerically computed and the results reveal that the electric field and chemical reaction enhances the transport (dispersion) of aerosols, i.e., the electric field removes efficiently the solid or liquid particles from gases discharged to the environment. Therefore, the results throw a light on aerosol removal mechanisms. Also, it is found that the transport of aerosols decreases with an increase in the porous parameter.

\section{Acknowledgement}

This work was supported by the University of Hong Kong through the Seed Funding Programme for Basic Research (Grant No. 200911159024).

\section{References}

[1] TAYLOR G. I. Dispersion of soluble matter in solvent flowing slowly through a tube[J]. Proc. Roy. Soc. Lond. A, 1953, 219(1137): 186-203.

[2] WATERMAN P. C. Matrix formulation of electromagnetic scattering $[\mathrm{J}]$. Proc. IEEE, 1965, 53(8): 805-812.

[3] WILliamS T. J., BAILEY A. G. Changes in the size distribution of a water-in-oil emulsion due to electricfield induced coalescence[J]. IEEE Trans. Ind. Appl., 1986, 22(3): 536-541.

[4] SCHMIDT J. J. Experimental study of electrocoalescence in a unit reactor[D]. Master Thesis, Austin, USA: University of Texas, 2000.

[5] SUN Lei, LIN Jian-zhong and BAO Fu-bing. Numerical simulation on the deposition of nanoparticles under laminar conditions $[\mathrm{J}]$. Journal of Hydrodynamics, 2006, 18(6): 676-680.

[6] GAN Fu-jun, LIN Jian-zhong and YU Ming-zhou. Particle size distribution in a planar jet flow undergoing shear-induced coagulation and breakage[J]. Journal of Hydrodynamics, 2010, 22(4): 445-455.

[7] YIN Zhao-qin, LIN Jian-zhong. Numerical simulation of the formation of nano-particles in an impinging twinjet[J]. Journal of Hydrodynamics, Ser. B, 2007, 19(6): 533-541.

[8] LIU Song, LIN Jian-zhong. Numerical simulation of nanoparticle coagulation in a Poiseuille flow via a moment method[J]. Journal of Hydrodynamics, 2008, 20(1): 1-9.
[10] JAYARATNE E. R., VERMA T. S. Environmental aerosols and their effect on the Earth's local fairweather electric field[J]. Meteorol. Atmos. Phys., 2004, 86(3-4): 275-280.

[11] RUDRAIAH N., DEVARAJU N. Effects of the reaction rate and the large size deformable aerosols on dispersion in atmospheric flow regarded as the turbulent fluid saturated porous media[J]. J. Porous Media, 2011, 14(3): 187-199.

[12] KENYON D. E. A. Mathematical model of water flux through aortic tissue[J]. Bull. Math. Bio., 1979, 41(1): 79-90.

[13] BOWEN R. M. Incompressible porous-media models by use of the theory of mixtures[J]. Int. J. Engg. Sci., 1980, 18(9): 1129-1148.

[14] BARRY S. I., PARKER K. H. and ALDIS G. K. Fluid flow over a thin deformable porous layer[J]. J. Appl. Math. Phys. (ZAMP), 1991, 42(5): 633-648.

[15] SAFFMAN P. G. On the boundary condition at surface of a porous medium[J]. Stud. in Appl. Math., 1971, 50(2): 93-101.

[16] RUDRAIAH N., NG C. O. Dispersion in porous media with and without reaction: A review[J]. J. Porous Media, 2007, 10(3): 219-248.

\section{Appendix}

$\Delta=\left(a_{0}^{2}+\alpha_{1}^{2} \sigma^{2}\right) \sinh a_{0}+2 a_{0} \alpha_{1} \sigma \cosh a_{0}$,

$\delta=a_{0} \sinh a_{0}+\alpha_{1} \sigma \cosh a_{0}$,

$c_{1}=\frac{W_{e}\left(\alpha-\alpha_{1} \sigma\right)}{\alpha_{1} \sigma R_{1}\left(\alpha^{2}-a_{0}^{2}\right)}+\frac{\varphi^{s}}{a_{0}^{2}}+\frac{a_{0}}{\Delta \alpha_{1} \sigma} \bullet$

$\left[\frac{W_{e}\left(\alpha_{1} \sigma-\alpha\right) \delta}{R_{1}\left(\alpha^{2}-a_{0}^{2}\right)}-\frac{\alpha_{1} \sigma \delta \varphi^{s}}{a_{0}^{2}}+\right.$

$\left.\frac{W_{e} \mathrm{e}^{-\alpha} \alpha_{1} \sigma\left(\alpha_{1} \sigma-\alpha\right)}{R_{1}\left(\alpha^{2}-a_{0}^{2}\right)}+\frac{\alpha_{1}^{2} \sigma^{2} \varphi^{s}}{a_{0}^{2}}\right]$,

$c_{2}=\frac{1}{\Delta}\left[\frac{W_{e}\left(\alpha_{1} \sigma-\alpha\right) \delta}{R_{1}\left(\alpha^{2}-a_{0}^{2}\right)}-\frac{\alpha_{1} \sigma \delta \varphi^{s}}{a_{0}^{2}}+\right.$

$\left.\frac{W_{e} \mathrm{e}^{-\alpha} \alpha_{1} \sigma\left(\alpha_{1} \sigma-\alpha\right)}{R_{1}\left(\alpha^{2}-a_{0}^{2}\right)}+\frac{\alpha_{1}^{2} \sigma^{2} \varphi^{s}}{a_{0}^{2}}\right]$,

$c_{3}=l_{2}+\frac{b_{0}\left(l_{1}-l_{2}\right)\left(\alpha_{2} \sigma \cos b_{0}-b_{0} \sin b_{0}\right)}{\left(\alpha_{2}^{2} \sigma^{2}-b_{0}^{2}\right) \sin b_{0}+2 b_{0} \alpha_{2} \sigma \cos b_{0}}$, 
256

$c_{4}=\frac{\left(c_{3}-l_{3}\right) \alpha_{2} \sigma}{b_{0}}$,
$l_{1}=\frac{\lambda R_{2} c_{1} a_{0} \sinh a_{0}}{R_{4}\left(a_{0}^{2}+b_{0}^{2}\right)}+\frac{\lambda R_{2} c_{2} a_{0} \cosh a_{0}}{R_{4}\left(a_{0}^{2}+b_{0}^{2}\right)}+$

$\frac{\alpha \mathrm{e}^{-\alpha} W_{e}}{\left(\alpha^{2}+b_{0}^{2}\right)}\left[\frac{\lambda R_{2}}{R_{1} R_{4}\left(\alpha^{2}-a_{0}^{2}\right)}-\frac{1}{R_{4}}\right]-\frac{\alpha_{2} \sigma \varphi^{f}}{b_{0}^{2}}+$

$\frac{\alpha_{2} \sigma \lambda R_{2} c_{1} \cosh a_{0}}{R_{4}\left(a_{0}^{2}+b_{0}^{2}\right)}+\frac{\alpha_{2} \sigma \lambda R_{2} c_{2} \sinh a_{0}}{R_{4}\left(a_{0}^{2}+b_{0}^{2}\right)}-$

$\frac{\alpha \sigma \mathrm{e}^{-\alpha} W_{e}}{\left(\alpha^{2}+b_{0}^{2}\right)}\left[\frac{\lambda R_{2}}{R_{1} R_{4}\left(\alpha^{2}-a_{0}^{2}\right)}-\frac{1}{R_{4}}\right]-\frac{\alpha_{2} \sigma \lambda R_{2} \varphi^{s}}{R_{4}\left(a_{0}^{2}+b_{0}^{2}\right)}$,

$l_{2}=\frac{-\lambda R_{2} c_{2} a_{0}}{\alpha_{2} \sigma R_{4}\left(a_{0}^{2}+b_{0}^{2}\right)}-\frac{\alpha W_{e}}{\alpha_{2} \sigma\left(a_{0}^{2}+b_{0}^{2}\right)}$.

$\left[\frac{\lambda R_{2}}{R_{1} R_{4}\left(\alpha^{2}-a_{0}^{2}\right)}-\frac{1}{R_{4}}\right]-\frac{\varphi^{f}}{b_{0}^{2}}+\frac{\lambda R_{2} \sigma c_{1}}{R_{4}\left(a_{0}^{2}+b_{0}^{2}\right)}-$

$\frac{W_{e}}{\alpha^{2}+b_{0}^{2}}\left[\frac{\lambda R_{2}}{R_{1} R_{4}\left(\alpha^{2}-a_{0}^{2}\right)}-\frac{1}{R_{4}}\right]-\frac{\lambda R_{2} \varphi^{s}}{R_{4}\left(a_{0}^{2} b_{0}^{2}\right)}$,

$l_{3}=\left(c_{4} b_{0}+\frac{\lambda R_{2} c_{2}}{2 a_{0} R_{4}}\right) \frac{1}{\alpha_{1} \sigma R_{1}\left(a_{0}^{2}+b_{0}^{2}\right)}-$

$\frac{\alpha W_{e}\left(\lambda R_{2}+R_{1}\right)}{\alpha_{1} \sigma R_{1} R_{4}\left(\alpha^{2}-a_{0}^{2}\right)\left(\alpha^{2}+b_{0}^{2}\right)}-\frac{c_{3}}{R_{1}\left(a_{0}^{2}+b_{0}^{2}\right)}-$

$\frac{\left(\lambda R_{2}+R_{1}\right) W_{e}}{R_{1} R_{4}\left(\alpha^{2}-a_{0}^{2}\right)^{2}\left(\alpha^{2}+b_{0}^{2}\right)}+\frac{\lambda R_{2} \varphi^{s}}{R_{4} a_{0}^{4} b_{0}^{2}}$

$l_{4}=\frac{1}{\Delta}\left\{\frac{b_{0}\left(c_{3} \sin b_{0}-c_{4} \cos b_{0}\right)}{R_{1}\left(a_{0}^{2}+b_{0}^{2}\right)}-\right.$

$\frac{\lambda R_{2}\left[\left(c_{1} a_{0}+c_{2}\right) \cosh a_{0}+\left(c_{1}+c_{2} a_{0}\right) \sinh a_{0}\right]}{2 a_{0} R_{1} R_{4}\left(a_{0}^{2}+b_{0}^{2}\right)}+$

$\frac{W_{e} \alpha \mathrm{e}^{-\alpha}\left(\lambda R_{2}-R_{1}\right)}{R_{1} R_{4}\left(\alpha^{2}-a_{0}^{2}\right)\left(\alpha_{0}^{2}-b_{0}^{2}\right)} \cdot$ $\frac{\alpha_{1} \sigma\left(c_{3} \cos b_{0}+c_{4} \sin b_{0}\right)}{R_{1}\left(a_{0}^{2}+b_{0}^{2}\right)}-$

$\frac{\alpha_{1} \sigma \lambda R_{2}\left(c_{1} \sinh a_{0}+c_{2} \cosh a_{0}\right)}{2 a_{0} R_{1} R_{4}\left(a_{0}^{2}+b_{0}^{2}\right)}-$

$\left.\frac{W_{e} \alpha_{1} \sigma \mathrm{e}^{-\alpha}\left[\lambda R_{2}-R_{1}\left(\alpha^{2}-a_{0}^{2}\right)\right]}{R_{1} R_{4}\left(\alpha^{2}-a_{0}^{2}\right)^{2}\left(\alpha^{2}+b_{0}^{2}\right)}\right\}$,

$\Delta_{11}=l_{2}+\frac{b_{0}\left(l_{1}-l_{2}\right)\left(\alpha_{2} \sigma \cos b_{0}-b_{0} \sin b_{0}\right)}{\left(\alpha_{2}^{2} \sigma^{2}-b_{0}^{2}\right) \sin b_{0}+2 b_{0} \alpha_{2} \sigma \cos b_{0}}$,

$\Delta_{12}=\frac{b_{0}\left(l_{1}-l_{2}\right)\left(\alpha_{2} \sigma \cos b_{0}-b_{0} \sin b_{0}\right)}{\left(\alpha_{2}^{2} \sigma^{2}-b_{0}^{2}\right) \sin b_{0}+2 b_{0} \alpha_{2} \sigma \cos b_{0}}$,

$\Delta_{13}=\frac{-\lambda R_{2} c_{1}}{R_{4}\left(a_{0}^{2}+b_{0}^{2}\right)}, \Delta_{14}=\frac{-\lambda R_{2} c_{2}}{R_{4}\left(a_{0}^{2}+b_{0}^{2}\right)}$,

$\Delta_{15}=\left[\frac{-\lambda R_{2}}{R_{1} R_{4}\left(\alpha^{2}+b_{0}^{2}\right)}-\frac{1}{R_{4}}\right] \frac{W_{e}}{\alpha^{2}+b_{0}^{2}}$,

$\Delta_{16}=\frac{\lambda R_{2} \varphi^{s}}{R_{4} a_{0}^{2} b_{0}^{2}}+\frac{\varphi^{f}}{b_{0}^{2}}$

$\Delta_{17}=\frac{\left(l_{4}-l_{3}\right) \alpha_{2} \sigma\left(a_{0} \sinh a_{0}+\alpha_{1} \sigma \cosh a_{0}\right)}{\left(a_{0}^{2}+\alpha_{1}^{2} \sigma^{2}\right) \sinh a_{0}+2 a_{0} \alpha_{1} \sigma \cosh a_{0}}$,

$\Delta_{18}=\frac{\lambda R_{2} c_{1}}{2 a_{0} R_{1} R_{4}\left(a_{0}^{2}+b_{0}^{2}\right)}, \quad \Delta_{19}=l_{3}+\frac{a_{0}}{\alpha_{1} \sigma} \Delta_{17}$,

$\Delta_{20}=\frac{\lambda R_{2} c_{2}}{2 a_{0} R_{1} R_{4}\left(a_{0}^{2}+b_{0}^{2}\right)}, \quad \Delta_{21}=\frac{c_{4}}{R_{1}\left(a_{0}^{2}+b_{0}^{2}\right)}$,

$\Delta_{22}=\frac{c_{3}}{R_{1}\left(a_{0}^{2}+b_{0}^{2}\right)}$,

$\Delta_{23}=\left[\frac{\lambda R_{2}}{R_{1} R_{4}\left(\alpha^{2}-a_{0}^{2}\right)^{2}}-\frac{1}{R_{4}\left(\alpha^{2}-a_{0}^{2}\right)}\right] \frac{W_{e}}{\alpha^{2}+b_{0}^{2}}$,

$\Delta_{24}=-\left(\frac{\lambda R_{2} \varphi^{s}}{R_{4} a_{0}^{4} b_{0}^{2}}+\frac{\varphi^{f}}{a_{0}^{2} b_{0}^{2}}\right), \Delta_{25}=l_{6}-\frac{\alpha_{2} \sigma \delta\left(l_{6}-l_{5}\right)}{\alpha_{2} \sigma \delta+b_{0}}$, 


$$
\begin{array}{ll}
\Delta_{29}=-\left[\frac{\lambda_{3}}{a_{0}^{2}+b_{0}^{2}}-\frac{2 a_{0} \lambda_{6}}{\left(a_{0}^{2}+b_{0}^{2}\right)}\right], \Delta_{30}=-\left(\frac{\lambda_{5}}{a_{0}^{2}+b_{0}^{2}}\right), & a_{17}=\frac{-W_{e}}{R_{1}\left(\alpha^{2}-a_{0}^{2}\right)}+\varepsilon \Delta_{23}, a_{18}=\frac{-\varphi^{s}}{a_{0}^{2}}+\varepsilon \Delta_{24}, \\
\Delta_{31}=-\left(\frac{\lambda_{4}}{a_{0}^{2}+b_{0}^{2}}-\frac{2 a_{0} \lambda_{5}}{\left(a_{0}^{2}+b_{0}^{2}\right)^{2}}\right), \Delta_{32}=-\frac{\lambda_{6}}{a_{0}^{2}+b_{0}^{2}}, & b_{11}=\Delta_{11}+\varepsilon \Delta_{25}, b_{12}=\varepsilon \Delta_{26}, b_{13}=\Delta_{12}+\varepsilon \Delta_{27}, \\
\Delta_{33}=-\frac{\lambda_{7}}{\alpha^{2}+b_{0}^{2}}, \Delta_{34}=-\frac{\lambda_{8}}{b_{0}^{2}}, & b_{17}=\Delta_{14}+\varepsilon \Delta_{31}, b_{18}=\varepsilon \Delta_{32}, \\
b_{19}=\Delta_{15}+\varepsilon \Delta_{33}, b_{20}=\Delta_{16}+\varepsilon \Delta_{34}, b_{16}=\varepsilon \Delta_{30},
\end{array}
$$

